\title{
Dielectric measurements in vanadium doped barium titanate
}

\author{
A HANUMAIAH, T BHIMASANKARAM, \\ S V SURYANARAYANA and G S KUMAR \\ Department of Physics, Osmania University, Hyderabad 500007, India
}

MS received 9 March 1994; revised 3 November 1994

\begin{abstract}
Ceramic samples of $\mathrm{Li}_{0.1} \mathrm{Ba}_{0.95}\left(\mathrm{~V}_{x} \mathrm{Ti}_{1-x}\right) \mathrm{O}_{3}$, where $x=0$ to 0.2 were prepared by solid state reaction method. The samples were characterized by XRD. The dielectric measurements have been carried out on the sintered disks as a function of the frequency $(100 \mathrm{~Hz}-1 \mathrm{MHz})$ and temperature $(300-420 \mathrm{~K})$. The variation of dielectric constant with temperature indicates a diffuse phase transition in all the samples studied.
\end{abstract}

Keywords. Dielectric; diffuse phase transition; doped barium titanate.

\section{Introduction}

The ferroelectrics with diffuse phase transition were first discovered in the solid solutions of barium titanate-barium tin oxide (Smolenskii and Isupov 1954). The broadening of the phase transition is a very common phenomena in solid solutions (Smolenskii 1970; Lines and Glass 1977) and other disorder structures. The ferroelectric--paraelectric phase transitions in these systems are not abrupt but they are gradual diffuse transitions occurring over a temperature range called Curie range. In this region the ferroelectrics exhibit unusual dielectric and ferroelectric characteristics. This behaviour is attributed to structural disorder and compositional fluctuations in the crystalline systems studied (Smolenskii 1984). The diffuse transitions do not occur at high temperatures where the defects are more mobile in the lattice. The presence of impurities also affect the phase transition temperatures of ferroelectric materials,

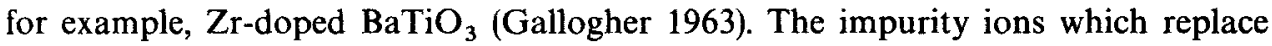
either $\mathrm{Ba}$ or $\mathrm{Ti}$ to some extent do show changes in dielectric constant and transition temperatures. In this present work $\mathrm{Li}_{0.1} \mathrm{Ba}_{0.95}\left(\mathrm{~V}_{x} \mathrm{Ti}_{1-x}\right) \mathrm{O}_{3}$ has been prepared and investigated for the variation of its dielectric constant with temperature and frequency.

\section{Experimental}

Lithium barium titanate $\mathrm{Li}_{0.1} \mathrm{Ba}_{0.95} \mathrm{TiO}_{3}$ and lithium barium vanadium titanate used in the present study were prepared by solid state double sintering method. Reagents of $\mathrm{Li}_{2} \mathrm{CO}_{3}, \mathrm{BaCO}_{3}, \mathrm{~V}_{2} \mathrm{O}_{5}$ and $\mathrm{TiO}_{2}$ were mixed in appropriate proportions to obtain $\mathrm{Li}_{0.1} \mathrm{Ba}_{0.95} \mathrm{~V}_{x} \mathrm{Ti}_{1-x} \mathrm{O}_{3}(x=0$ to 0.2$)$. The components were homogeneously mixed, ground to a fine powder and calcined at $1050^{\circ} \mathrm{C}$ for $2 \mathrm{~h}$. The presintered mixture was then ground, mixed with $2 \%$ poly vinyl alcohol as a binder, pressed into pellets of diameter $11 \mathrm{~mm}$ and thickness $2 \mathrm{~mm}$, using a pressure of $1 \times 10^{9} \mathrm{~N} / \mathrm{m}^{2}$. The pellets were initially kept at $400^{\circ} \mathrm{C}$ for $2 \mathrm{~h}$ to eliminate the binder and were sintered at $1150^{\circ} \mathrm{C}$ for $1 \mathrm{~h}$ in a linearly programmable furnace. Lithium in the place of barium has been used to lower the sintering temperature of $\mathrm{BaTiO}_{3}$ (Subba Rao et al 1981). Using a JEOL diffractometer and $\mathrm{FeK}_{\alpha}$ radiation, the XRD patterns of all the samples were 


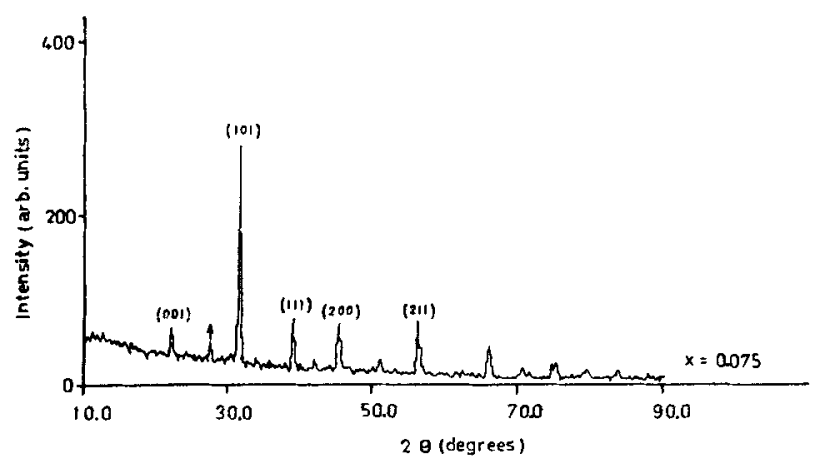

Figure 1. X-ray diffractogram of lithium barium vanadium titanate $(x=0.075)$.

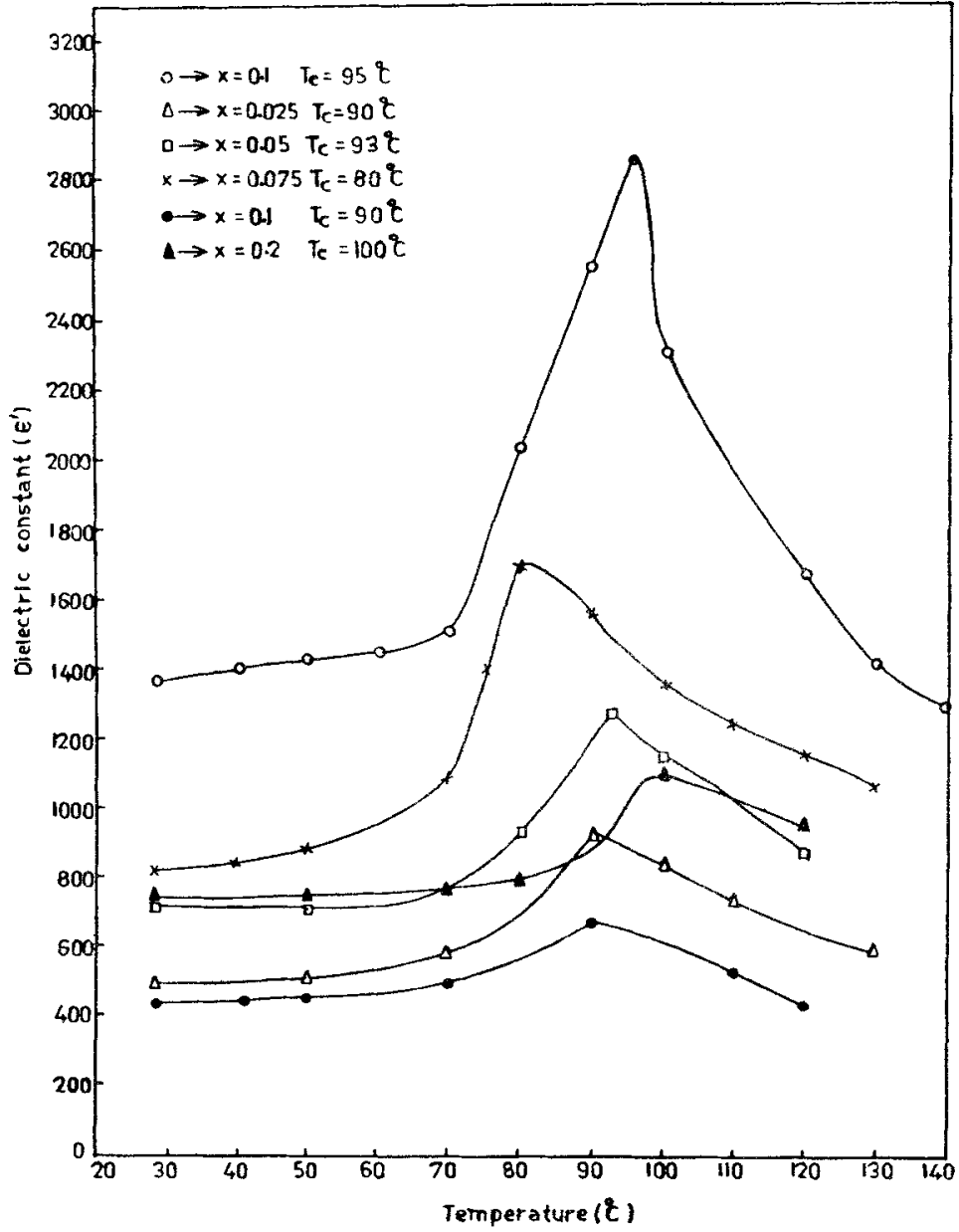

Figure 2. The variation of dielectric constant $\left(\varepsilon^{\prime}\right)$ with temperature at $1 \mathrm{kHz}$ frequency $(0, x=0.0 ; \triangle, x=0.025, \square, x=0.05 ; x, x=0.075 ; \ominus, x=0.1 ; \Delta, x=0.2)$. 
recorded employing a scan speed of $4 \mathrm{deg} / \mathrm{min}$. The dependence of $\varepsilon$ in all the samples was measured employing HP 4192A impedance analyser as a function of temperature from ambient to $150^{\circ} \mathrm{C}$.

\section{Results}

The XRD pattern shown in figure 1 for sample with $x=0.075$ indicated the formation of the single phase with tetragonal symmetry but there was one peak (marked in figure 1) which could not be identified to any particular phase. This peak could not be identified with any peak of $\mathrm{V}_{2} \mathrm{O}_{5}$. A similar type of XRD pattern was observed in other samples, however, the intensity of unidentified peak increased with the increase of vanadium content. The lattice parameters of the samples were computed and found to be almost equal to that of barium titanate.

The density of the sample determined by loss of weight method indicated that as the concentration of vanadium increased, the density was found to decrease. The variation of $\varepsilon^{\prime}$ with temperature is presented in figure 2. As the concentration of vanadium was increased the Curie temperature $T_{c}$ was found to decrease from $100^{\circ} \mathrm{C}-80^{\circ} \mathrm{C}$. It was also noticed that there was a fall of peak value $\varepsilon_{\mathrm{c}}^{\prime}$ with vanadium content. The transitions are not found to be sharp as can be seen in figure 2. This type of behaviour of $\varepsilon^{\prime}$ is associated with diffuse phase transition (DPT). Such transition is not associated invariably with Curie-Weiss law and an alternative expression was suggested by Uchino and Nomura (1982) which is given as

$$
1 / \varepsilon^{\prime}=1 / \varepsilon_{\mathrm{c}}^{\prime}+\left[\left(T-T_{\mathrm{c}}\right)^{2} / 2 \varepsilon_{\mathrm{c}}^{\prime} \delta^{2}\right]
$$

where $\varepsilon_{\mathrm{c}}^{\prime}$ and $\delta$ are maximum dielectric constant and intensity of DPT respectively. From the knowledge of $\varepsilon_{\mathrm{c}}^{\prime}, T_{\mathrm{c}}$, and $\varepsilon^{\prime}$, the value of $\delta$ can be computed in the present case. The value of $\delta$ thus computed is given in table 1 . The diffuse transition is more pronounced in samples with higher vanadium concentration. As $\varepsilon=\varepsilon^{\prime}-j \varepsilon^{\prime \prime}$, the $\varepsilon^{\prime \prime}$ data are plotted as a function of temperature at $1 \mathrm{kHz}$ frequency (figure 3 ). The results are identical with those obtained by Issac Robin et al (1992) in the case of lithium barium zirconate titanate system.

The variation of dielectric constant with temperature at different frequencies for the sample $x=0.1$ is shown in figure 4 . We observed that the $T_{\mathrm{c}}$ for all the frequencies is the same and not much of dispersion occurs in the frequency range studied. Therefore it can be assumed that the sample behaviour resembles to that of a relaxor ferroelectric.

Table 1. Dielectric data of lithium barium vanadium titanate $\left(\mathrm{Li}_{0 \cdot 1} \mathrm{Ba}_{0 \cdot 95} \mathrm{~V}_{x} \mathrm{Ti}_{1-x} \mathrm{O}_{3}\right)$.

\begin{tabular}{lcrrrr}
\hline $\boldsymbol{x}$ & Density $(\mathrm{g} / \mathrm{cc})$ & $\varepsilon_{\boldsymbol{R} T}^{\prime}$ & \multicolumn{1}{c}{$\varepsilon_{\mathrm{c}}^{\prime}$} & \multicolumn{1}{c}{$T_{\mathrm{c}}$} & $\delta$ \\
\hline 0.0 & 5.58 & 1364 & 2854 & 95 & 27 \\
0.025 & $5 \cdot 58$ & 491 & 926 & 90 & 29 \\
0.05 & 5.44 & 720 & 1280 & 93 & 37 \\
0.075 & 5.37 & 810 & 1700 & 80 & 28 \\
0.1 & $5 \cdot 35$ & 425 & 670 & 90 & 28 \\
0.2 & $5 \cdot 33$ & 740 & 1100 & 100 & 32 \\
\hline
\end{tabular}




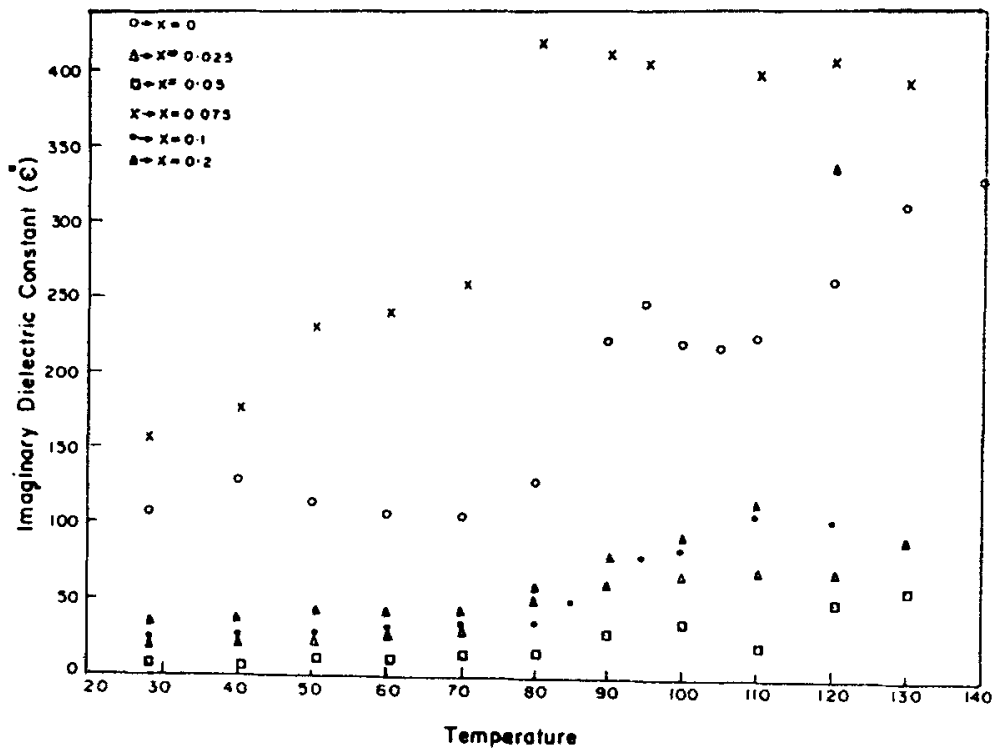

Figure 3. The variation of imaginary dielectric constant $\left(\varepsilon^{\prime \prime}\right)$ with temperature at $1 \mathrm{kHz}$ frequency. $(0, x=0.0 ; \Delta, x=0.025, \square, x=0.05 ; x, x=0.075 ; 0, x=0.1 ; \Delta, x=0 \cdot 2)$.

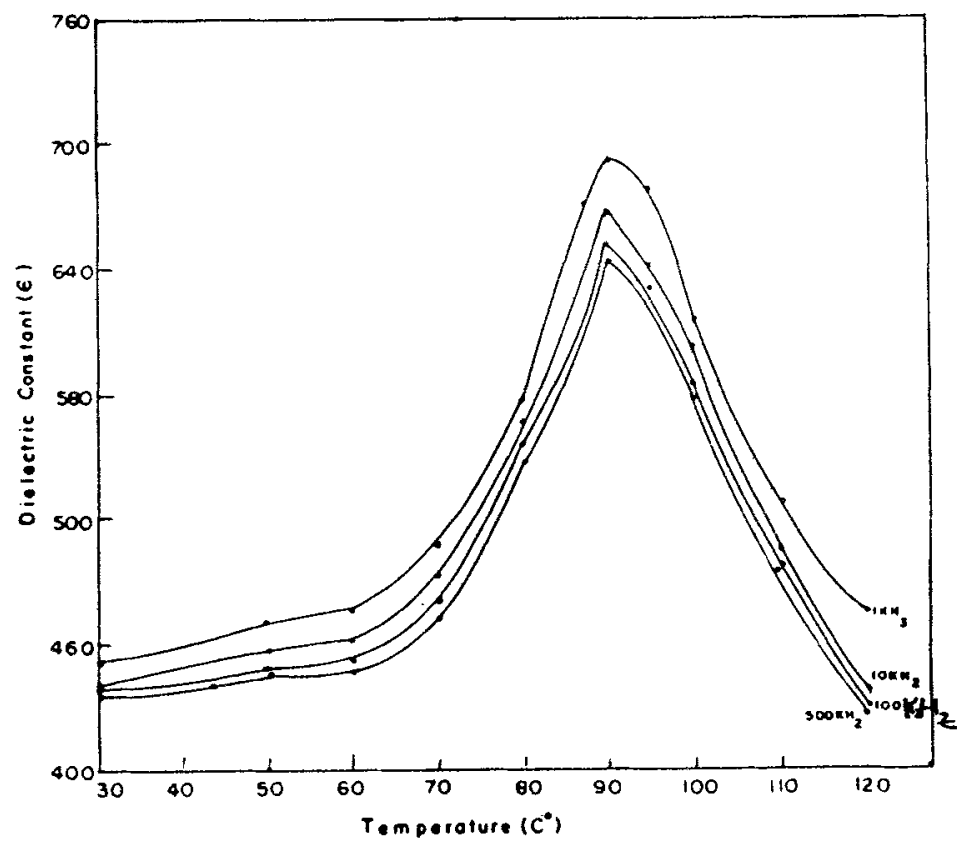

Figure 4. The variation of dielectric constant $\left(\varepsilon^{\prime}\right)$ with temperature at different frequencies $(x=0 \cdot 1)$. 


\section{Acknowledgement}

The authors thank the University Grants Commission, New Delhi, for financial assistance through a sponsored project.

\section{References}

Gallogher 1963 J. Am. Ceram. Soc. 46359

Issac Robin A, Prasad Rao A V and Sambasiva Rao K 1992 Indian J. Pure Appl. Phys. 30267

Lines M E and Glass A M 1977 Principles and applications of ferroelectrics and related materials (Oxford: Clarendon Press) p. 285

Smolenskii G A and Isupov V A 1954 Dokl. Akad. Nauk. SSSR 97653

Smolenskii G A 1970 J. Phys. Soc. Jap. S28 26

Smolenskii G A 1984 Ferroelectrics 53129

Subba Rao P S V, Sambasiva Rao K and Bhanumati A 1981 J. Mater. Sci. Lett. 6389

Uchino K and Nomura S 1982 Ferroelectrics Lett. Sect. 4455 